\title{
Survival outcomes of hepatectomy for stage B Hepatocellular carcinoma in the BCLC classification
}

Toshiya Kamiyama1*, Tatsuya Orimo ${ }^{1}$, Kenji Wakayama', Shingo Shimada', Akihisa Nagatsu', Hideki Yokoo', Hirofumi Kamachi ${ }^{1}$, Kenichiro Yamashita ${ }^{2}$, Tsuyoshi Shimamura ${ }^{3}$ and Akinobu Taketomi ${ }^{1}$

\begin{abstract}
Background: Because hepatectomy is not recommended in patients with stage B hepatocellular carcinoma (HCC) of the Barcelona Clinic Liver Cancer (BCLC) staging, we evaluated the survival outcomes of hepatectomy for stage $B$ in the BCLC system.

Methods: Data were collected from 297 consecutive adult stage B patients who underwent curative hepatectomy for HCC between 1996 and 2014 in Hokkaido University Hospital. Overall survival (OS), disease-free survival (DFS), and risk factors were analyzed using the Kaplan-Meier method. Independent prognostic factors were evaluated using a Cox proportional hazards regression model. AP-factor (alpha-fetoprotein [AFP] $\times$ protein induced by vitamin $\mathrm{K}$ absence or antagonism factor II [PIVKA-II]) was categorized according to the serum concentrations of AFP and PIVKA-II: AP1 (AFP $<200 \mathrm{ng} / \mathrm{ml}$ and PIVKA- $\|<100 \mathrm{mAU} / \mathrm{ml})$, AP2 (AFP $\times$ PIVKA- $\|<10^{5}$ ), and AP3 (AFP $\times$ PIVKA- $-1 \geq 10^{5}$ ).
\end{abstract}

Results: There were 130 deaths among our 297 stage B patients (43.8\%). The causes of death in these cases were HCC recurrence $(n=106 ; 81.5 \%)$, liver failure $(n=7 ; 5.4 \%)$, and other causes $(n=17 ; 16.1 \%)$. The operative mortality rate was $0.34 \%$ (1/297). The 5-year OS and DFS rates for the stage B cases were 54.3 and $21.9 \%$, respectively. By multivariate analysis, tumor number and AP-factor were risk factors for both survival and recurrence that were tumor related and could be evaluated preoperatively. The study patients with stage B HCC were classified into three groups by tumor number (B1, 1; B23, 2 or 3; B4over: $\geq 4$ ) and into three groups stratified by AP-factor (AP1, AP2, and AP3). The 5-year OS rates of B1, B23, and B4over were 63.6, 52.3, and 29.0\%. The 5-year OS rates of AP1, AP2, and AP3 were 67.6, 65.2, and 39.1\%. Stratified by the 5-year OS rate, stage B HCC patients were classified into three subgroups (A-C).The 5-year OS rates of groups $\mathrm{A}$ (B1 or $\mathrm{B} 23$ and $\mathrm{AP}-1$ or $\mathrm{AP}-2), \mathrm{B}$ (B1 or $\mathrm{B} 23$ and $\mathrm{AP}-3$, or $\mathrm{B} 4$ over and $\mathrm{AP}-1$ or $\mathrm{AP}-2)$, and $\mathrm{C}$ (B4over and AP-3) were 69.5, 43.7, and 21.3\%.

Conclusion: Stage B HCC patients with a tumor number $\leq 3$ and/or AP-factor $<1 \times 10^{5}$ show acceptable 5 -year OS rates and could be treated by hepatectomy.

Keywords: Hepatocellar carcinoma, Hepatectomy, BCLC staging.

\footnotetext{
*Correspondence: t-kamiya@med.hokudai.ac.jp

'Department of Gastroenterological Surgery I, Hokkaido University Graduate

School of Medicine, North 15, West 7, Kita-ku, Sapporo 060-8638, Japan

Full list of author information is available at the end of the article
} 


\section{Background}

Hepatectomy for hepatocellular carcinoma (HCC) has the highest local controllability of all local treatments for this disease and achieves a good survival rate [1, 2]. However, according to the Barcelona Clinic Liver Cancer (BCLC) staging classification [3], hepatic resection is contraindicated in intermediate-stage patients (stage $\mathrm{B}$ HCC) and only transarterial chemoembolization (TACE) is indicated [3]. In contrast, some patients with stage $B$ $\mathrm{HCC}$, according to the BCLC staging classification, are indicated for hepatectomy according to a Japanese evidence-based treatment algorithm [4] and a consensusbased treatment algorithm for HCC proposed by the Japan Society of Hepatology (JSH algorithm) [5], with acceptable prognoses [1]. Thus, the indication for hepatectomy differs among several staging systems.

Moreover, stage B HCC in the BCLC staging classification shows considerable heterogeneity because this stage includes tumor-related factors, namely, up to three tumors where at least one is $>3 \mathrm{~cm}$ in diameter, more than three tumors of any size, or single tumors exceeding $5 \mathrm{~cm}$ in diameter. These classifications of the BCLC staging classification, JSH algorithm, and Japanese evidence-based treatment algorithm rely on tumor morphologic factors and not biomarkers such as alphafetoprotein (AFP) and protein induced by vitamin $K$ absence or antagonism factor II (PIVKA-II).

In this present study, we analyzed the survival outcomes of consecutive patients who underwent primary curative hepatectomy for HCC at our center to identify the factors related to the prognosis and recurrence of stage B HCC in the BCLC staging system and newly classify this stage according to the factors identified. Using our new classification, we sought to evaluate the indications for, and significance of, performing a hepatectomy for a stage B HCC as defined by the BCLC staging system.

\section{Methods \\ Patients}

Between May 1996 and October 2014, 776 consecutive adult patients underwent hepatectomy for $\mathrm{HCC}$ at our center. Patients were classified into five groups in accordance with the BCLC staging system: stage 0 $(n=55)$, stage A $(n=327)$, stage $B(n=297)$, and stage $\mathrm{C}(n=97)$. These patients were selected according to our algorithm, which incorporates the indocyanine green retention rate at $15 \mathrm{~min}$ (ICGR15) and remnant liver volume to determine the operative indication [6]. TACE was preoperatively performed in $44(14.8 \%)$ patients, percutaneous ethanol injection therapy in $2(0.7 \%)$, radiofrequency ablation in $3(1 \%)$. As we previously reported [7], AP-factor $($ AFP $\times$ PIVKA-II) was categorized into three groups according to the serum concentrations of AFP and PIVKA-II, i.e., AP1 (AFP < $200 \mathrm{ng} / \mathrm{ml}$ and PIVKA-II $<100 \mathrm{mAU} / \mathrm{ml})$, AP2 $\left(\right.$ AFP $\times$ PIVKA-II $\left.<10^{5}\right)$, and AP3 (AFP $\times$ PIVKA-II $\left.\geq 10^{5}\right)$.

All analyses in our present study were performed in accordance with the ethical guidelines of Hokkaido University Hospital. This study was approved by the Institutional Review Board of Hokkaido University.

\section{Hepatectomy}

Anatomical resection is defined as complete anatomical removal of lesion(s) on the basis of Couinaud's classification (segmentectomy, sectionectomy, and hemihepatectomy or extended hemihepatectomy) in patients with sufficient functional reserve. Non-anatomical partial but complete resection was achieved in all patients. $\mathrm{R} 0$ resections were performed in all stage $\mathrm{B}$ patients, and all resection surfaces were found to be histologically free of $\mathrm{HCC}$.

\section{HCC recurrence}

For the first 2 years after hepatectomy, patients underwent follow-up evaluation every 3 months comprising liver function tests, measurements of AFP and PIVKAII, US, and dynamic computed tomography (CT). After 2 years, routine $\mathrm{CT}$ was performed once every 4 months. If recurrence was suspected, $\mathrm{CT}$ and magnetic resonance imaging (MRI) were performed, as well as CT angiography and bone scintigraphy, if necessary. This enabled precise determination of the site, number, size, and extent of invasiveness of the recurrent lesions.

\section{Statistical analysis}

The overall survival (OS) and disease-free survival (DFS) rates were calculated using the Kaplan-Meier method and compared between groups using the log-rank test. Potential prognostic factors were identified by univariate analysis using the log-rank test. Independent prognostic factors were evaluated using a Cox proportional hazards regression model. In this study, $p<0.05$ was considered significant. Statistical analyses were performed using JMP (version 12 for Windows; SAS Institute, Cary, NC).

\section{Results \\ Demographics of stage B patients}

The mean age of the patients with HCC Stage B was 63.7 years (range, $18-89$ years). Of the 297 patients with Stage B disease, 253 (85.2\%) were male, 105 (35.4\%) were hepatitis B virus surface antigen-positive, and 87 (29.3\%) were hepatitis $C$ virus antibody-positive. In addition, 290 (97.6\%) were categorized as Child-Pugh class A and 67 (22.6\%) was diagnosed as liver cirrhosis pathologically. The tumors of $33(11.1 \%)$ patients was limited to one segment, those of $83(27.9 \%)$ to one section, those of $131(44.1 \%)$ in two sections, those of 35 (11.8\%) to three 
sections, and those of 15 (5.1\%) involving more than three sections. Non-anatomical partial resection was performed in $56(18.9 \%)$ patients. Anatomical resection of segmentectomy, sectionectomy, and hemihepatectomy/ extended hemihepatectomy were performed in 27 (9\%), $64(21.5 \%)$, and $150(50.5 \%)$ patients, respectively. The patients were followed up for a median of 98.3 months (range, 6.8-226.8 months). Twenty one patients $(0.098 \%)$ were lost to follow-up for more than 5 years from the last confirmed date.

\section{Sites of HCC recurrence and treatment}

Of the 297 stage B patients analyzed, 211 (71.0\%) showed recurrence. Of the 94 recurrent cases from group A, 59 (62.8\%) had recurrence only in the liver and 35 at extrahepatic sites (including or excluding the liver). Of the 99 recurrent cases in group B, 47 (47.5\%) had recurrence only in the liver and 52 at extrahepatic sites. Of the 18 recurrent cases of group C, 9 (50.0\%) had recurrence only in the liver and 9 in extrahepatic sites. Although there were no significant differences among the groups, recurrence in group A patients tended to occur only in the liver, whereas it tended to occur in extrahepatic sites in group B and C patients $(p=0.095)$.

We treated $\mathrm{HCC}$ recurrence by repeated hepatectomy $(n=21)$, transcatheter arterial chemoembolization $(n=135)$, local ablation $(n=12)$, and resection of extrahepatic metastasis $(n=19)$.

\section{Survival outcomes and causes of death}

The 5-year OS rates for the stage $0, \mathrm{~A}, \mathrm{~B}$, and $\mathrm{C} \mathrm{HCC}$ cases were $85.8,76.3,54.3$, and $19.0 \%$, respectively. The corresponding 5-year DFS rates were 47.1, 38.2, 21.9, and $10.2 \%$, respectively. There were 130 deaths among our 297 stage B patients (43.8\%). The causes of death in these cases were HCC recurrence $(n=106 ; 81.5 \%)$, liver failure $(n=7 ; 5.4 \%)$, and other causes $(n=17 ; 16.1 \%)$. The operative mortality rate was $0.34 \%(1 / 297)$.

\section{Univariate and multivariate analyses of survival rates}

By univariate analysis, significant risk factors for the reduced survival of stage $B$ patients included serum albumin level, AFP, PIVKA-II, AP-factor, anatomical resection, blood loss, tumor number, microscopic portal vein invasion, and a noncancerous liver (Table 1). The significant risk factors for recurrence were serum albumin level, PIVKA-II, AP-factor, a higher tumor number, microscopic portal vein invasion, and microscopic hepatic vein invasion (Table 1). Multivariate analysis by the Cox proportional hazard model was then performed using these significant factors to further clarify the risk factors for lower survival and $\mathrm{HCC}$ recurrence. The significant factors identified by univariate analysis for stage B HCC patient survival were included in multivariate analysis which showed that the lower serum albumin level $(p=0.0024)$, nonanatomical resection $(p=0.0132)$, AP-factor (AP2 vs AP3, $p=0.0023)$, tumor number $(1$ vs $\geq 4$, $p=0.0001 ; 2$ or 3 vs $\geq 4, p=0.0011)$ and microscopic portal vein invasion (vp0 vs vp2, $p=0.0001$; vp1 vs $\mathrm{vp} 2, p=0.0133)$ were independent risk factors for survival (Table 2). The significant factors identified by univariate analysis for stage B HCC patient recurrence were included in multivariate analysis which showed that the lower serum albumin level $(p=0.026)$, PIVKA-II $(100-1000$ vs $>1000, p=0.038 ; 100-1000$ vs $\leq 100, p=0.002$ ), AP-factor (AP1 vs AP2, $p=0.0036$; AP2 vs AP3, $p=0.001)$, tumor number ( 1 vs 2 or $3, p=0.037 ; 1$ vs $\geq 4, p<0.001)$ and microscopic portal vein invasion (vp0 vs vp2, $p=0.006)$ (Table 3).

\section{Overall and disease-free survival outcomes categorized by tumor number and AP-factor}

The study patients with stage B HCC were classified into three groups by tumor number (B1, 1; B23, 2 or 3; B4over: 24 ) and into three groups stratified by AP-factor (AP1, AP2, and AP3). This grouping was done because the HCC tumor number and AP-factor were found to be risk factors for poorer survival and recurrence by multivariate analysis and these are tumor-related factors that can be evaluated preoperatively. The 5-year OS rates for B1, B23, and B4over cases were 63.6, 52.3, and 29.0\%, respectively. There was a significant difference between each of these groups $(p<0.01)$ except between B1 and B23. There was no difference in OS between the tumor number 2 and $3(p=0.8224)$. The 5 -year DFS rates for B1 and B23 were 30.1 and $15.5 \%$, respectively $(p<0.01)$ (Fig. 1a). The 5-year OS rates for the AP1, AP2, and AP3 cases were $67.6,65.2$, and $39.1 \%$, respectively. There was a significant difference between AP1 and AP3 $(p<0.01)$ and between AP2 and AP3 $(p<0.01)$. The 5year DFS rates for the AP1, AP2, and AP3 patients were $23.8,28.6$, and $15.2 \%$, respectively. There were also significant differences between AP1 and AP3 $(p<0.01)$ and between AP2 and AP3 ( $p<0.01$; Fig. 1b).

\section{Stage B patients stratified by tumor number and AP-factor}

Stage $B$ patients were further categorized in nine ways by combining tumor number and AP-factor which are tumor-related factors that can be evaluated preoperatively. The 5-year OS rates of these nine categories ranged from 21.3 to $75.6 \%$ (Table 4). Stratified by the 5 year OS rate, stage B HCC patients were classified into three subgroups (A-C). Group A included patients with a tumor number $\leq 3$ and AP-factor $<1 \times 10^{5}$, group B included patients with tumor number $\leq 3$ and APfactor $>1 \times 10^{5}$ or tumor number $\geq 4$ and AP- 
Table 1 Univariate analysis of variables predictive (clinical and tumor associated factors) for stage B HCC patient survival and recurrence

\begin{tabular}{|c|c|c|c|c|}
\hline Variables & & $n$ & $\begin{array}{l}p \text { value } \\
\text { Survival }\end{array}$ & $\begin{array}{l}p \text { value } \\
\text { Recurrence }\end{array}$ \\
\hline \multirow{2}{*}{ Sex } & A17l & 253 & & \\
\hline & Female & 44 & & \\
\hline \multirow[t]{2}{*}{ Age } & $<60$ & 117 & 0.9306 & 0.4414 \\
\hline & $\geq 60$ & 180 & & \\
\hline \multirow[t]{2}{*}{ HBV } & Negative & 191 & 0.0516 & 0.2386 \\
\hline & Positive & 105 & & \\
\hline \multirow[t]{2}{*}{$\mathrm{HCV}$} & Negative & 210 & 0.7523 & 0.2126 \\
\hline & Positive & 87 & & \\
\hline \multirow[t]{2}{*}{ Albumin (g/dl) } & $<4$ & 139 & 0.0006 & 0.0439 \\
\hline & $\geq 4$ & 158 & & \\
\hline \multirow{2}{*}{$\begin{array}{l}\text { Total bilirubin } \\
(\mathrm{mg} / \mathrm{dl})\end{array}$} & $<0.8$ & 160 & 0.2827 & 0.4807 \\
\hline & $\geq 0.8$ & 137 & & \\
\hline \multirow[t]{2}{*}{ ICGR15 (\%) } & $<15$ & 178 & 0.3471 & 0.1136 \\
\hline & $\geq 15$ & 119 & & \\
\hline \multirow[t]{3}{*}{ Tumor number } & 1 & 145 & $<0.0001^{*} 1$ & $<0.0001{ }^{*} 2$ \\
\hline & $20 r 3$ & 100 & & \\
\hline & $\geq 4$ & 52 & & \\
\hline \multirow[t]{3}{*}{ Tumor size $(\mathrm{cm})$} & $\leq 3$ & 8 & 0.9998 & 0.7932 \\
\hline & $3-5$ & 54 & & \\
\hline & $\geq 5$ & 235 & & \\
\hline \multirow{2}{*}{$\begin{array}{l}\text { Anatomical } \\
\text { resection }\end{array}$} & Yes & 241 & 0.0182 & 0.2306 \\
\hline & No & 56 & & \\
\hline \multirow[t]{2}{*}{ Blood loss (ml) } & $<1100$ & 239 & 0.0027 & 0.1937 \\
\hline & $\geq 1100$ & 58 & & \\
\hline \multirow[t]{3}{*}{ AFP $(n g / m l)$} & $\leq 200$ & 201 & $0.0028^{*} 3$ & 0.1276 \\
\hline & 200-1000 & 26 & & \\
\hline & $>1000$ & 70 & & \\
\hline \multirow[t]{3}{*}{ PIVKA-II (mAU/ml) } & $\leq 100$ & 82 & $0.0354^{*} 4$ & $0.0048{ }^{*} 5$ \\
\hline & $100-1000$ & 75 & & \\
\hline & $>1000$ & 139 & & \\
\hline \multirow{3}{*}{$\begin{array}{l}\text { AP-factor } \\
\text { (AFP*PIVKA-II) }\end{array}$} & AP1 & 65 & $<0.0001^{*} 6$ & $0.0009 * 7$ \\
\hline & AP2 & 99 & & \\
\hline & AP3 & 133 & & \\
\hline \multirow[t]{4}{*}{ Differentiation } & Well & 19 & 0.2092 & 0.1655 \\
\hline & Moderate & 160 & & \\
\hline & Poor & 115 & & \\
\hline & Unknown & 19 & & \\
\hline \multirow{4}{*}{$\begin{array}{l}\text { Microscopic } \\
\text { portal vein } \\
\text { invasion }\end{array}$} & vp0 & 205 & $0.0003^{*} 8$ & $0.0103 * 9$ \\
\hline & vp1 & 62 & & \\
\hline & vp2 & 28 & & \\
\hline & Unknown & 1 & & \\
\hline
\end{tabular}

Table 1 Univariate analysis of variables predictive (clinical and tumor associated factors) for stage B HCC patient survival and recurrence (Continued)

\begin{tabular}{lllll}
\hline $\begin{array}{l}\text { Microscopic } \\
\text { hepatic vein }\end{array}$ & vv0 & 271 & $0.01463^{*} 10$ & $0.0262^{*} 11$ \\
invasion & vv1 & 25 & & \\
& Unknown & 1 & & \\
$\begin{array}{l}\text { Non-cancerous } \\
\text { liver }\end{array}$ & Cirrhosis & 67 & 0.1328 & 0.1772 \\
& Non-cirrhosis & 230 & & \\
\hline
\end{tabular}

HbsAg, hepatitis B virus s antigen; HCV, anti-hepatitis C virus antibody; AFP, alpha-fetoprotein; PIVKA-II, protein induced by vitamin $\mathrm{K}$ absence or antagonism factor II; ICGR15, indocyanine green retention rate at 15 min; AP-factor, a product of the serum levels of AFP and PIVKA-II. HCC patients were classified into three groups: AP1 (AFP $<200 \mathrm{ng} / \mathrm{ml}$ and PIVKA-II $<100 \mathrm{mAU} / \mathrm{ml}$ ), AP2 $\left(\right.$ AFP $\times$ PIVKA-II $\left.<10^{5}\right)$, and AP3 (AFP $\times$ PIVKA-II $\geq 10^{5}$ )

vp0: no tumor thrombus in the portal vein

vp1: tumor thrombus distal to the second branches of the portal vein vp2: tumor thrombus in the second branches of the portal vein vv0: no tumor thrombus in the hepatic vein

vv1: tumor thrombus in a branch of the hepatic vein

When the subgroups were more than three, $p$-value in Table 1 was showed a significant difference as a group. $P$-value between the subgroups was showed with asterisk

${ }^{*} 1$ : tumor number 1 vs $\geq 4(p<0.0001)$, tumor number 2 or 3 vs $\geq 4(p=0.0003)$

*2: tumor number 1 vs 2 or3 ( $p=0.0047)$, tumor number 1 vs $\geq 4(p<0.0001)$, tumor number $20 r 3$ vs $\geq 4(p=0.0122)$

* 3 : AFP $\leq 200$ vs $200-1000(p=0.0072), \leq 200$ vs $>1000(p=0.0009)$

* 4 : PIVKA-II $\leq 100$ vs $>1000(p=0.0043)$

*5: PIVKA-II $\leq 100$ vs $100-1000(p=0.0009), \leq 100$ vs $>1000(p=0.0224)$

*6: AP-1 vs Ap-3 $(p=0.0001)$, AP-2 vs Ap-3 $(p<0.0001)$

*7: AP-1 vs Ap-3 $(p=0.0012)$, AP-2 vs Ap-3 $(p=0.0074)$

8: vp0 vs vp1 $(p=0.0005)$, vp0 vs vp2 $(p<0.0001)$

*9: vp0 vs vp2 $(p=0.0024)$

*10: vv0 vs vv1 $(p=0.0123)$

*11: vv0 vs vv1 $(p=0.0181)$

factor $<1 \times 10^{5}$, and group $\mathrm{C}$ incorporated patients with tumor number $\geq 4$ and AP-factor $>1 \times 10^{5}$. The 5-year PS rates of groups A-C were 69.5, 43.7, and 21.3\%, respectively. There were significant differences between each of these groups $(p<0.01)$. The 5 -year DFS of groups A and B were 30.5 and $14.3 \%$, respectively $(p<0.01$; Fig. 2).

Table 2 Multivariate analysis of variables predictive for stage B HCC patient survival

\begin{tabular}{lllll}
\hline Survival & $p$ value & Risk ratio & \multicolumn{2}{c}{$\begin{array}{l}\text { 95\% confidence } \\
\text { interval }\end{array}$} \\
\hline Albumin (g/dl), <4 vs $\geq 4$ & 0.0024 & 1.837 & 1.241 & 2.728 \\
Anatomical vs nonanatomical & 0.0132 & 0.509 & 0.304 & 0.866 \\
AP-factor, AP2 vs AP3 & 0.0023 & 0.416 & 0.234 & 0.732 \\
Tumor number, 1 vs 4over & 0.0001 & 0.380 & 0.229 & 0.615 \\
$\quad$ 2,3 vs $\geq 4$ & 0.0011 & 0.414 & 0.248 & 0.699 \\
$\begin{array}{c}\text { Microscopic portal vein } \\
\text { invasion, vp0 vs vp2 }\end{array}$ & 0.0001 & 0.302 & 0.174 & 0.545 \\
$\quad$ & 0.0133 & 0.435 & 0.231 & 0.837 \\
\hline
\end{tabular}

PIVKA-II, protein induced by vitamin $\mathrm{K}$ absence or antagonism factor II; AP-factor, a product of the serum levels of AFP and PIVKA-II

vp0: no tumor thrombus in the portal vein

vp1: tumor thrombus distal to the second branches of the portal vein

vp2: tumor thrombus in the second branches of the portal vein 
Table 3 Multivariate analysis of variables predictive for stage B HCC patient recurrence

\begin{tabular}{lllll}
\hline Recurrence & $p$ value & Risk ratio & \multicolumn{2}{c}{$\begin{array}{l}\text { 95\% confidence } \\
\text { interval }\end{array}$} \\
\hline Albumin $(\mathrm{g} / \mathrm{dl}),<4$ vs $\geq 4$ & 0.026 & 1.382 & 1.040 & 1.834 \\
$\begin{array}{l}\text { PIVKA-II }(\mathrm{mAU} / \mathrm{ml}) \text {, } \\
\leq 100 \text { vs }>1000\end{array}$ & 0.437 & 0.537 & 0.266 & 0.983 \\
$100-1000$ vs $>1000$ & 0.038 & 1.479 & 1.023 & 2.127 \\
$100-1000$ vs $\leq 100$ & 0.002 & 2.755 & 1.447 & 5.717 \\
AP-factor, AP1 vs AP2 & 0.036 & 2.102 & 1.049 & 4.548 \\
$\quad$ AP2 vs AP3 & 0.001 & 0.562 & 0.395 & 0.795 \\
Tumor number, 1 vs 2,3 & 0.037 & 0.709 & 0.514 & 0.979 \\
$\quad 1$ vs $\geq 4$ & $<0.001$ & 0.485 & 0.326 & 0.739 \\
Microscopic portal vein & 0.006 & 0.488 & 0.310 & 0.805 \\
invasion, vp0 vs vp2 & & & &
\end{tabular}

PIVKA-II, protein induced by vitamin $\mathrm{K}$ absence or antagonism factor II; AP-factor, a product of the serum levels of AFP and PIVKA-II vp0: no tumor thrombus in the portal vein

vp1: tumor thrombus distal to the second branches of the portal vein vp2: tumor thrombus in the second branches of the portal vein

\section{Discussion}

In our present study, univariate and multivariate analysis was used to identify risk factors in patients with BCLC stage B HCC. Of the risk factors identified for both survival and recurrence, tumor number and AP-factor can be determined preoperatively. Our stage B patients were categorized into nine groupings according to these two risk factors. Based on the 5-year OS rates among these nine categories, stage $B$ patients were classified into three subgroups (A-C) with 5-year OS rates of 69.5, 43.7, and $21.3 \%$, respectively. Even in stage B HCC cases, the 5-year OS rates of some patients with a tumor number $\leq 3$ and/or AP-factor $<1 \times 10^{5}$ ranged from 43.7 to $69.5 \%$. These patients could therefore be treated with hepatectomy.

The main limit of BCLC staging is the considerable prognostic heterogeneity within each stage. Stage B HCC under this system is composed of a heterogeneous population because the classifications are done using tumor status, liver function, and physical status [3]. In recent years, Bolondi et al. [8] and Kudo et al. [9] proposed modified models of the BCLC staging system for prognostic prediction in HCC patients. However, because these suggested subclassifications were
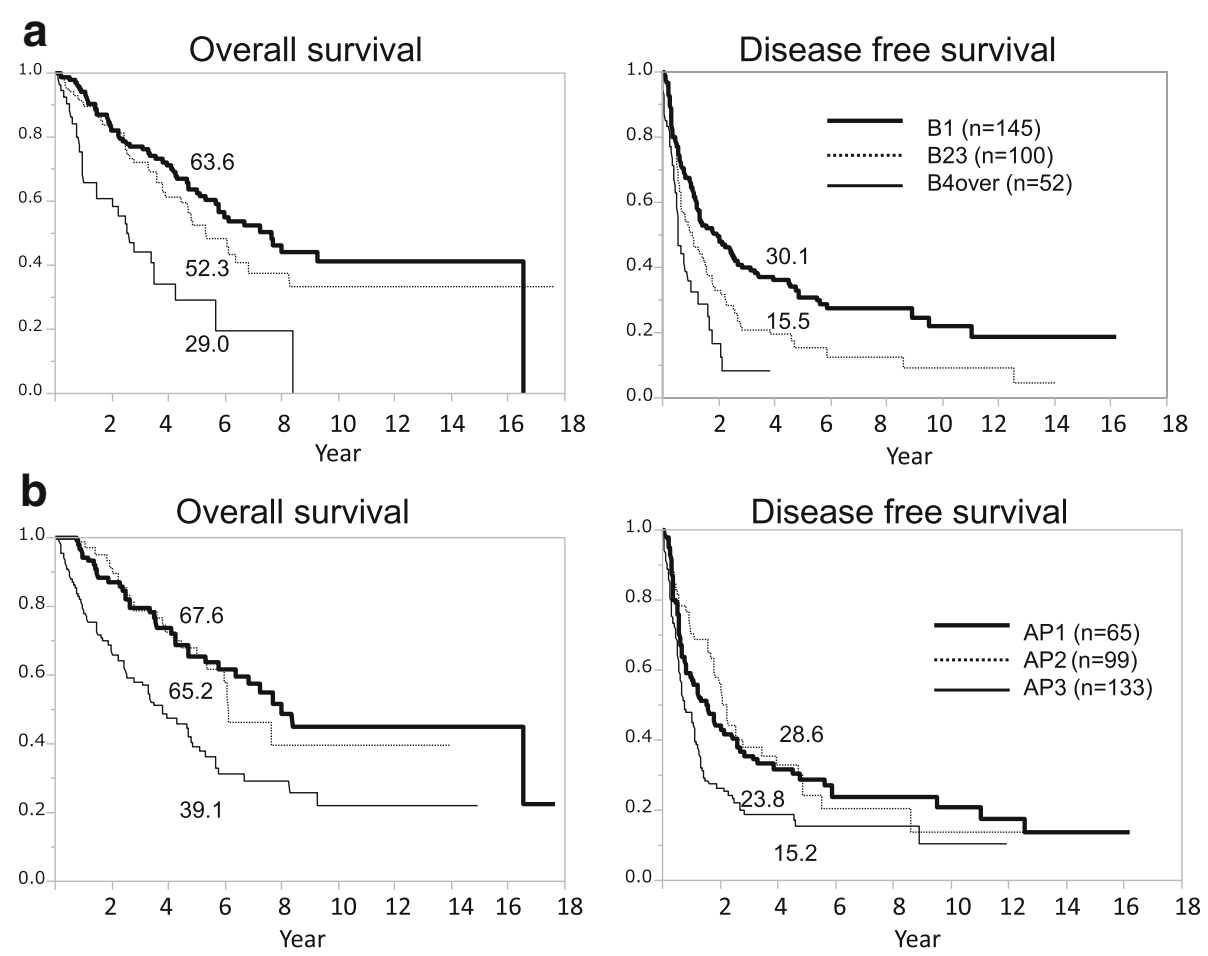

Fig. 1 Overall survival curves according to tumor number and AP-factor. a The 5-year overall survival rates of B1, B23, and B4over were 63.6, 52.3, and 29.0\%, respectively. There was a significant difference between each of these groups $(p<0.01)$, except for B1 and B23. The 5-year disease-free survival rates of B1 and B23 were 30.1 and 15.5\%, respectively $(p<0.01)$. b The 5 -year overall survival rates of AP1, AP2, and AP3 were 67.6, 65.2, and $39.1 \%$, respectively. There was a significant difference between AP1 and AP3 $(p<0.01)$ and between AP2 and AP3 $(p<0.01)$. The 5-year disease-free survival rates of AP1, AP2, and AP3 were 23.8, 28.6, and 15.2\%, respectively. There was a significant difference between AP1 and AP3 $(p<0.01)$ and between AP2 and AP3 $(p<0.01)$ 
Table 4 Categorization of patients into nine categories by tumor number and AP-factor and subclassification of BCLC stage B HCC

\begin{tabular}{lllll}
\hline & AP-1 & AP-2 & AP-3 & $(n)$ \\
\hline B1 & $75.6 \%$ & $73.7 \%$ & $45.3 \%$ & $(145)$ \\
& $(35)$ & $(53)$ & $(57)$ & \\
B23 & $57.1 \%$ & $65.6 \%$ & $43.0 \%$ & $(100)$ \\
& $(25)$ & $(27)$ & $(48)$ & \\
B4over & $60.0 \%$ & $34.7 \%$ & $21.3 \%$ & $(52)$ \\
& $(5)$ & $(19)$ & $(28)$ & \\
(n) & $(65)$ & $(99)$ & $(133)$ & $(297)$ \\
\hline
\end{tabular}

AP-factor, a product of the serum levels of AFP and PIVKA-II. HCC patients were classified into three groups: AP1 (AFP $<200 \mathrm{ng} / \mathrm{ml}$ and PIVKA-II $<100$ $\mathrm{mAU} / \mathrm{ml})$, AP2 (AFP $\times$ PIVKA-II $<10^{5}$ ), and AP3 (AFP $\times$ PIVKA-II $\geq 10^{5}$ ). B1, B23, and $B 4$ over were classified by tumor numbers 1,2 or 3 , and $\geq 4$, respectively. Group A comprises B1 or B23 and AP-1 or AP-2. Group B comprises B1 or B23 and AP-3, or B4over and AP-1 or AP-2. Group C comprises B4over and AP-3

conducted to decide on the best TACE treatment strategy, they were limited in helping clinicians with hepatectomy-related treatment decisions. Wada et al. [10] reported on the selection criteria for hepatic resection in BCLC stage B HCC. However, their subclassification relied on tumor number and size and not on tumor markers. The indications for a large HCC should also be reconsidered due to reports on the biological malignancy of solitary large HCC. It has been proposed that solitary large HCC should be considered a specific subtype that is less malignant than nodular HCC, because the expression levels of some human genes closely related to invasion and metastasis are significantly lower in these lesions [11]. It has also been reported that after resection of solitary large HCC, the clinical outcomes are similar to those of small HCC but are significantly better than those in patients with multiple HCC [12]. This study and the report by Torzilli G [13] included large solitary $\mathrm{HCC}$ (above $5 \mathrm{~cm}$ in diameter) as BCLC B HCC. Therefore, the classification of solitary tumors above $5 \mathrm{~cm}$ should be characterized as BCLC B by the staging system.

Given the aforementioned issues, a reliable biomarker to determine the biological malignancy of HCC must be included in the current staging system. AFP and PIVKAII have been used previously as useful tumor markers of HCC and are associated with a poor prognosis after hepatectomy $[14,15]$. AFP is related to tumor differentiation [16], whereas PIVKA-II is related to vascular invasion $[17,18]$. Although the latter is the most influential factor for recurrence and survival in patients undergoing hepatectomy [19], the oncological implications of AFP and PIVKA-II remain unclear. We previously established the AP-factor, a product of the serum levels of AFP and PIVKA-II, as a critical marker for preoperatively predicting tumor malignancy [7]. In the present study, tumor number and AP-factor were identified as the tumorrelated risk factors for survival and recurrence by multivariate analysis that could be evaluated preoperatively. We could clearly categorize our BCLC stage B patients into three subgroups by tumor number and AP-factor; the 5-year OS rates of these subgroups (A-C) were 69.5, 43.7, and 21.3\%, respectively. Notably, Yamakado et al. previously reported a 5-year overall survival rate of $23.7 \%$ in BCLC B patients treated with TACE [20]. Hence, the operative success of groups A and B was acceptable. Moreover, Kim et al. reported that after propensity score matching in BCLC B patients, the 1-, 2-, 3-, and 5-year overall survival rates in the resection group were $90,88,75$, and $63 \%$, compared with $79,48,35$, and $22 \%$ in the no-surgery group whom $94 \%$ had TACE as the first treatment $(P<0.001)[21]$.

Because approximately $90 \%$ of patients with HCC also have hepatitis $B$ and/or hepatitis $C$, and thus have chronic hepatitis or cirrhosis [22], the liver functional reserve was decreased in almost all of our current study patients. Liver resection in cirrhotic patients is thus
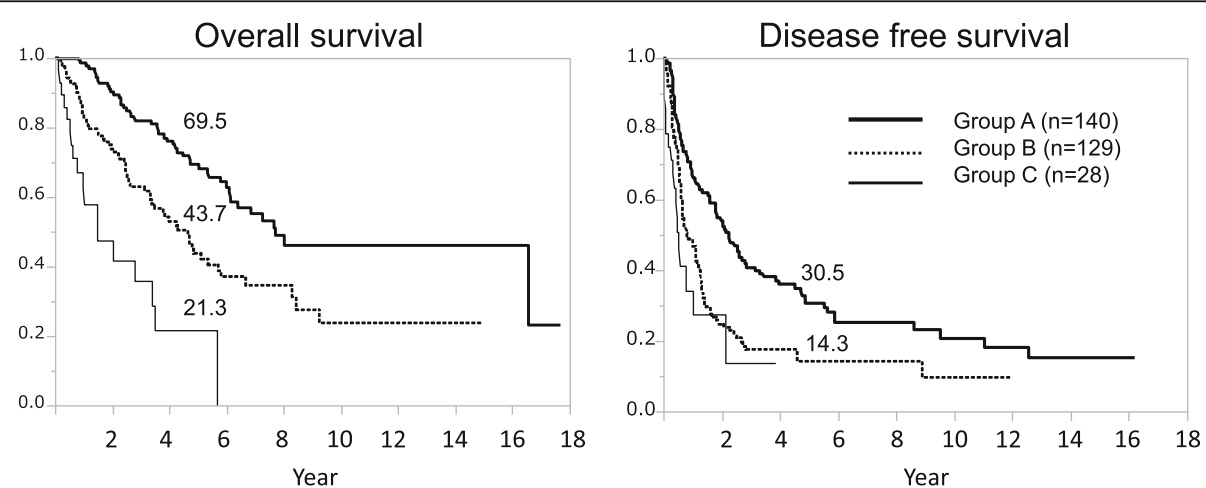

Fig. 2 Overall survival curves for groups A, B and C. Overall survival curves for groups A-C with 5-year patient survival rates of 69.5, 43.7, and 21.3\%, respectively. There was a significant difference between each of these groups $(p<0.01)$. The 5 -year disease-free survival rates of group $A$ and group $B$ were 30.5 and $14.3 \%$, respectively $(p<0.01)$ 
associated with high mortality rates (between 8.9 and 19.6\%) [23]. Conversely, recent advances in surgical techniques and pre- and postoperative care, including the decision criteria for hepatectomy and indications for liver resection, have been applied to extended hepatectomy [6, 24]. These preoperative evaluations allow a major hepatectomy to be more safely performed, although operative mortality is never completely avoided, even in donor hepatectomy for living donor liver transplantation [25]. In our present study, the operative mortality rates were as low as $0.39 \%$. Moreover, the large multicentric survey of Torzilli $\mathrm{G}$ and colleagues reported a 90 -day mortality rate of $2.7 \%$ and a 5 -year overall survival rate of $61 \%$ for BCLC B patients who underwent surgery [13]. Because hepatectomy is not as invasive and does not impose a large burden on patients from the point of view of operative mortality, we contend that the indications for hepatectomy could be expanded in BCLC stage B HCC cases.

Hepatectomy is associated with a high risk of recurrence. Thus, it has been proposed that salvage transplantation be considered after hepatectomy in HCC patients with preserved liver function [26, 27]. Recurrence patterns after curative hepatectomy have been analyzed previously in patients classified according to the Milan criteria [28]. In that study, about $30 \%$ of the patients whose lesions exceeded the Milan criteria did not develop HCC recurrence. About half of the studies patients with HCC recurrence met the Milan criteria, representing about $30 \%$ of those who exceeded the Milan criteria. HCC that was even beyond the Milan criteria was considered an indication for salvage transplantation. This further supports our belief that hepatectomy could be indicated for HCC stage B.

\section{Conclusions}

In conclusion, the 5-year overall survival rates of patients with tumor number $\leq 3$ and/or APfactor $<1 \times 10^{5}$ were acceptable even in BCLC stage B HCC patients. These patients could be treated with hepatectomy. Hepatectomy could also be performed after careful consideration in HCC patients with a tumor number $\geq 4$ and AP-factor $>1 \times 10^{5}$.

\footnotetext{
Abbreviations

AFP: Alpha-fetoprotein; AP-factor: AFP × PIVKA-II; BCLC: Barcelona Clinic Liver Cancer; CT: Computed tomography; DFS: Disease-free survival;

HCC: Hepatocellular carcinoma; ICGR15: Indocyanine green retention rate at 15 min; MRI: Magnetic resonance imaging; OS: Overall survival; PIVKA-

II: Protein induced by vitamin $\mathrm{K}$ absence or antagonism factor II;

TACE: Transarterial chemoembolization
}

\section{Acknowledgements}

The authors thank the staff of Gastroenterological Surgery I, Graduate School of Medicine, Hokkaido University, for their kind co-operation with this study.
Funding

Not applicable.

Availability of data and materials

All data generated or analyzed during this study are included in this published article.

\section{Authors' contributions}

TK was in charge of the study conception and design. TO, KW, SS, HK, and KY contributed to the acquisition of data. TK, AN, HY, TS, and AT contributed to the analysis and interpretation of data. TK drafted the manuscript. All authors read and approved the final manuscript.

\section{Ethics approval and consent to participate}

All analyses in our present study were performed in accordance with the ethical guidelines of Hokkaido University Hospital. This study was approved by the Institutional Review Board of Hokkaido University. The study was performed according to the Helsinki Declaration guidelines.

\section{Consent for publication}

We obtained consent for publication from the patients.

\section{Competing interests}

The authors declare that they have no competing interests.

\section{Publisher's Note}

Springer Nature remains neutral with regard to jurisdictional claims in published maps and institutional affiliations.

\section{Author details}

${ }^{1}$ Department of Gastroenterological Surgery I, Hokkaido University Graduate School of Medicine, North 15, West 7, Kita-ku, Sapporo 060-8638, Japan. ${ }^{2}$ Department of Transplantation Surgery, Hokkaido University Graduate School of Medicine, Sapporo, Japan. ${ }^{3}$ Department of Organ Transplantation, Hokkaido University Hospital, Sapporo, Japan.

Received: 5 June 2017 Accepted: 13 August 2017

Published online: 22 August 2017

\section{References}

1. Arii S, Yamaoka Y, Futagawa S, Inoue K, Kobayashi K, Kojiro M, Makuuchi M, Nakamura Y, Okita K, Yamada R. Results of surgical and nonsurgical treatment for small-sized hepatocellular carcinomas: a retrospective and nationwide survey in Japan. The liver cancer study Group of Japan. Hepatology. 2000:32(6):1224-9.

2. Hasegawa K, Kokudo N, Imamura H, Matsuyama Y, Aoki T, Minagawa M, Sano K, Sugawara Y, Takayama T, Makuuchi M. Prognostic impact of anatomic resection for hepatocellular carcinoma. Ann Surg. 2005; 242(2):252-9.

3. Tremosini S, Reig M, de Lope CR, Forner A, Bruix J. Treatment of early hepatocellular carcinoma: towards personalized therapy. Dig Liver Dis. 2010; 42(Suppl 3):S242-8.

4. Kokudo N, Makuuchi M. Evidence-based clinical practice guidelines for hepatocellular carcinoma in Japan: the J-HCC quidelines. J Gastroenterol. 2009;44(Suppl 19):119-21.

5. Kudo M, Izumi N, Kokudo N, Matsui O, Sakamoto M, Nakashima O, Kojiro M, Makuuchi M, Hepatology HCCEPoJSo. Management of hepatocellular carcinoma in Japan: consensus-based clinical practice guidelines proposed by the Japan Society of Hepatology (JSH) 2010 updated version. Dig Dis. 2011:29(3):339-64

6. Kamiyama T, Nakanishi K, Yokoo H, Kamachi H, Tahara M, Yamashita K, Taniguchi M, Shimamura T, Matsushita M, Todo S. Perioperative management of hepatic resection toward zero mortality and morbidity: analysis of 793 consecutive cases in a single institution. J Am Coll Surg. 2010;211(4):443-9.

7. Kamiyama T, Yokoo H, Kakisaka T, Orimo T, Wakayama K, Kamachi H, Tsuruga Y, Yamashita K, Shimamura T, Todo S, et al. Multiplication of alphafetoprotein and protein induced by vitamin $\mathrm{K}$ absence-II is a powerful predictor of prognosis and recurrence in hepatocellular carcinoma patients after a hepatectomy. Hepatol Res. 2015;45(10):E21-31. 
8. Bolondi L, Burroughs A, Dufour JF, Galle PR, Mazzaferro V, Piscaglia F, Raoul $J$, Sangro B. Heterogeneity of patients with intermediate (BCLC B) Hepatocellular carcinoma: proposal for a subclassification to facilitate treatment decisions. Semin Liver Dis. 2012;32(4):348-59.

9. Kudo M, Arizumi T, Ueshima K, Sakurai T, Kitano M, Nishida N. Subclassification of BCLC B stage Hepatocellular carcinoma and treatment strategies: proposal of modified Bolondi's subclassification (Kinki criteria). Dig Dis. 2015;33(6):751-8.

10. Wada H, Eguchi H, Noda T, Ogawa H, Yamada D, Tomimaru Y, Tomokuni A, Asaoka T, Kawamoto K, Gotoh K, et al. Selection criteria for hepatic resection in intermediate-stage (BCLC stage B) multiple hepatocellular carcinoma. Surgery. 2016;160(5):1227-35.

11. Wang W, Yang LY, Huang GW, Lu WQ, Yang ZL, Yang JQ, Liu HL. Genomic analysis reveals RhoC as a potential marker in hepatocellular carcinoma with poor prognosis. B J Cancer. 2004;90(12):2349-55.

12. Yang LY, Wang W, Peng JX, Yang JQ, Huang GW. Differentially expressed genes between solitary large hepatocellular carcinoma and nodular hepatocellular carcinoma. World J Gastroenterol. 2004;10(24):3569-73.

13. Torzilli G, Belghiti J, Kokudo N, Takayama T, Capussotti L, Nuzzo G, Vauthey JN, Choti MA, De Santibanes E, Donadon M, et al. A snapshot of the effective indications and results of surgery for hepatocellular carcinoma in tertiary referral centers: is it adherent to the EASL/AASLD recommendations?: an observational study of the HCC east-west study group. Ann Surg. 2013;257(5):929-37.

14. Imamura H, Matsuyama $Y$, Miyagawa $Y$, Ishida $K$, Shimada R, Miyagawa S, Makuuchi M, Kawasaki S. Prognostic significance of anatomical resection and des-gamma-carboxy prothrombin in patients with hepatocellular carcinoma. Br J Surg. 1999;86(8):1032-8.

15. Shimada M, Takenaka K, Fujiwara Y, Gion T, Kajiyama K, Maeda T, Shirabe K, Sugimachi K. Des-gamma-carboxy prothrombin and alpha-fetoprotein positive status as a new prognostic indicator after hepatic resection for hepatocellular carcinoma. Cancer. 1996;78(10):2094-100.

16. Tamura S, Kato T, Berho M, Misiakos EP, O'Brien C, Reddy KR, Nery JR, Burke GW, Schiff ER, Miller J, et al. Impact of histological grade of hepatocellular carcinoma on the outcome of liver transplantation. Arch Surg. 2001;136(1): 25-30. discussion 31

17. Toyoda H, Kumada T, Kiriyama S, Sone Y, Tanikawa M, Hisanaga Y, Yamaguchi A, Isogai M, Kaneoka Y, Washizu J. Prognostic significance of simultaneous measurement of three tumor markers in patients with hepatocellular carcinoma. Clin Gastroenterol Hepatol. 2006;4(1):111-7.

18. Inoue S, Nakao A, Harada A, Nonami T, Takagi H. Clinical significance of abnormal prothrombin (DCP) in relation to postoperative survival and prognosis in patients with hepatocellular carcinoma. Am J Gastroenterol. 1994;89(12):2222-6.

19. Izumi R, Shimizu K, li T, Yagi M, Matsui O, Nonomura A, Miyazaki !. Prognostic factors of hepatocellular carcinoma in patients undergoing hepatic resection. Gastroenterology. 1994;106(3):720-7.

20. Yamakado K, Miyayama S, Hirota S, Mizunuma K, Nakamura K, Inaba Y, Maeda H, Matsuo K, Nishida N, Aramaki T, et al. Subgrouping of intermediate-stage (BCLC stage B) hepatocellular carcinoma based on tumor number and size and child-Pugh grade correlated with prognosis after transarterial chemoembolization. Jpn J Radiol. 2014;32(5):260-5.

21. Kim H, Ahn SW, Hong SK, Yoon KC, Kim HS, Choi YR, Lee HW, Yi NJ, Lee KW, Suh KS, et al. Survival benefit of liver resection for Barcelona clinic liver cancer stage B hepatocellular carcinoma. Br J Surg. 2017;104(8):1045-52.

22. Ikai I, Arii S, Okazaki M, Okita K, Omata M, Kojiro M, Takayasu K, Nakanuma Y, Makuuchi M, Matsuyama Y, et al. Report of the 17th Nationwide follow-up survey of primary liver cancer in Japan. Hepatol Res. 2007;37(9):676-91.

23. Moser MA, Kneteman NM, Minuk GY. Research toward safer resection of the cirrhotic liver. HPB Surg. 2000;11(5):285-97.

24. Makuuchi M, Kosuge T, Takayama T, Yamazaki S, Kakazu T, Miyagawa S, Kawasaki S. Surgery for small liver cancers. Semin Surg Oncol. 1993;9(4): 298-304.

25. Hashikura Y, Ichida T, Umeshita K, Kawasaki S, Mizokami M, Mochida S, Yanaga K, Monden M, Kiyosawa K, Japanese Liver Transplantation S. Donor complications associated with living donor liver transplantation in Japan. Transplantation. 2009;88(1):110-4.

26. Adam R, Azoulay D, Castaing D, Eshkenazy R, Pascal G, Hashizume K, Samuel $D$, Bismuth $H$. Liver resection as a bridge to transplantation for hepatocellular carcinoma on cirrhosis: a reasonable strategy? Ann Surg. 2003;238(4):508-18. discussion 518-509
27. Belghiti J, Cortes A, Abdalla EK, Regimbeau JM, Prakash K, Durand F, Sommacale D, Dondero F, Lesurtel M, Sauvanet A, et al. Resection prior to liver transplantation for hepatocellular carcinoma. Ann Surg. 2003;238(6): 885-92. discussion 892-883

28. Kamiyama T, Nakanishi K, Yokoo H, Kamachi H, Tahara M, Suzuki T, Shimamura T, Furukawa H, Matsushita M, Todo S. Recurrence patterns after hepatectomy of hepatocellular carcinoma: implication of Milan criteria utilization. Ann Surg Oncol. 2009;16(6):1560-71.

\section{Submit your next manuscript to BioMed Central and we will help you at every step:}

- We accept pre-submission inquiries

- Our selector tool helps you to find the most relevant journal

- We provide round the clock customer support

- Convenient online submission

- Thorough peer review

- Inclusion in PubMed and all major indexing services

- Maximum visibility for your research

Submit your manuscript at www.biomedcentral.com/submit
) Biomed Central 